\title{
Hyperprolactinemia and infertility: new insights
}

\author{
Ursula B. Kaiser, MD \\ Division of Endocrinology, Diabetes and Hypertension, Brigham and Women's Hospital, Boston, Massachusetts, USA.
}

\begin{abstract}
Clinical vignette: A 29-year-old woman is referred for management of infertility. After menarche at age 12, menses occurred irregularly for a year and then became regular. She initiated use of oral contraceptive pills at the age of 18, then stopped at age 27 to try to conceive. Evaluation revealed hyperprolactinemia with serum prolactin of $90 \mathrm{ng} / \mathrm{ml}$; pituitary MRI showed a 6-mm microadenoma. Other pituitary function tested was normal. Therapy was initiated with bromocriptine, but it was poorly tolerated, with fatigue, nausea, and lightheadedness to the point of syncopal events during her work as a hairdresser. Treatment was changed to cabergoline, with similar difficulties. Prolactin levels declined to the $30 \mathrm{~s}-40 \mathrm{~s}$, but she was never able to tolerate the medication sufficiently to attain normal prolactin levels, and menses were sporadic and infrequent, with only $2-3$ occurring per year. She and her husband had not conceived despite regular unprotected intercourse. She asks whether other medical treatment options might be available for her infertility.
\end{abstract}

\section{Current therapy}

Prolactin-secreting adenomas are the most common functioning pituitary tumor $(1,2)$. In women, most prolactinomas are microadenomas $(<10 \mathrm{~mm})$, and hypersecretion of prolactin leads to amenorrhea, galactorrhea, and infertility. Similarly, men may also have hypogonadism and infertility. Infertility results from suppression of gonadotropin secretion, manifest as hypogonadotropic hypogonadism $(\mathrm{HH})(3)$. The goals of therapy are to normalize prolactin, reduce tumor size, reverse hypogonadism, and restore fertility. The most common treatment approach is with the dopamine receptor agonists, bromocriptine and cabergoline. Bromocriptine normalizes prolactin and decreases tumor size in $80 \%-90 \%$ of patients with microadenomas. Adverse effects include nausea, orthostatic hypotension, headache, and fatigue. The more selective D2 receptor agonist, cabergoline, is more effective and better tolerated than bromocriptine. While cabergoline is the most commonly used medical therapy and can be used in women trying to conceive, bromocriptine has been used more extensively during pregnancy and as a result its safety record is better established. Dopamine receptor agonist intolerance leading to discontinuation occurs in $10 \%-20 \%$ of patients.

Conflict of interest: The author holds equity in ExpressScripts, Immunogen, and Synageva. A member of her family has been employed in the past calendar year by Synageva and Genzyme and is a member of the Board of Directors of Immunogen.

Citation for this article: J Clin Invest. 2012;122(10):3467-3468. doi:10.1172/JCI64455.

\section{Knowledge gaps}

While hyperprolactinemia is a well-established cause of $\mathrm{HH}$ and infertility, the mechanisms of these effects are not well understood. Elevated prolactin may impact reproduction through inhibitory effects on hypothalamic gonadotropin-releasing hormone $(\mathrm{GnRH})$ neurons and/or on the pituitary gland to reduce secretion of the gonadotropins luteinizing hormone (LH) and follicle-stimulating hormone $(\mathrm{FSH})$, resulting in a reduction in both amplitude and frequency of LH pulses (4). Prolactin may act directly on GnRH neurons to suppress GnRH secretion, or the effects may be indirect, mediated through other afferent pathways, perhaps via other neurons influencing GnRH release (5). During lactation, additional metabolic factors induced by negative energy balance may also contribute to disruption of pulsatile $\mathrm{GnRH}$ and LH secretion.

In this issue of the JCI, a study by Sonigo et al. (6) using a mouse model of hyperprolactinemia provides important insights into the neural pathways by which the hypothalamic-pituitary-gonadal axis is suppressed to cause infertility. Continuous infusion of prolactin subcutaneously in female mice resulting in hyperprolactinemia (mean, $260 \mathrm{ng} / \mathrm{ml}$ ) is associated with loss of estrous cyclicity, anovulation as reflected by a decrease in ovarian corpora lutea, a reduction in circulating $\mathrm{LH}$ and FSH levels, and a decrease in pituitary $L b b$ and Fshb mRNA. Kisspeptin, a neuropeptide

\section{Research advances}

encoded by the Kiss 1 gene, has been recognized in recent years as a potent stimulus of GnRH secretion, of critical importance for pubertal maturation and regulation of reproductive function (7). In the mouse, kisspeptin is expressed in two hypothalamic neuronal populations: in the arcuate nucleus (ARC), which mediates sex steroid negative feedback regulation of GnRH release, and in the anteroventral periventricular nucleus (AVPV), which mediates the estrogen-induced ovulatory surge of GnRH in females. Kisspeptin immunoreactivity was reduced in both the ARC and AVPV in the hyperprolactinemic mouse model, suggesting that kisspeptin plays a role in mediating hyperprolactinemia-induced anovulation and $\mathrm{HH}$. In support of this hypothesis, the administration of intraperitoneal injections of kisspeptin once daily into the hyperprolactinemic mice for 20 days restored estrous cyclicity, induced ovulation, and increased pituitary $L b b$ and $F s h b$ mRNA and circulating LH and FSH levels. Furthermore, treatment of ex vivo medial basal hypothalamic explants with prolactin suppressed $\mathrm{GnRH}$ release, whereas cotreatment with kisspeptin and prolactin prevented the prolactin-induced decrease. Taken together with a prior report that kisspeptin neurons express prolactin receptors (5), these data suggest that kisspeptin may be the missing link between hyperprolactinemia and the associated $\mathrm{HH}$ and infertility (Figure 1).

\section{Implications and future directions}

The study by Sonigo et al. (6) provides important new insights into potential mechanisms and pathways mediating hypogonadotropic anovulation associated with hypeprolactinemia, which results in amenorrhea, infertility, and hypogonadism. The data suggest that suppression of kisspeptin may mediate prolactin-induced inhibition of cycles, although other GnRH neuromodulators may also contribute to these effects, as may direct effects on GnRH neurons or on pituitary gonadotropes (Figure 1). The ability of exogenously administered kisspeptin to reverse the hypogonadotropic effects of hyperprolactinemia has potential therapeutic implications. Treatment with 


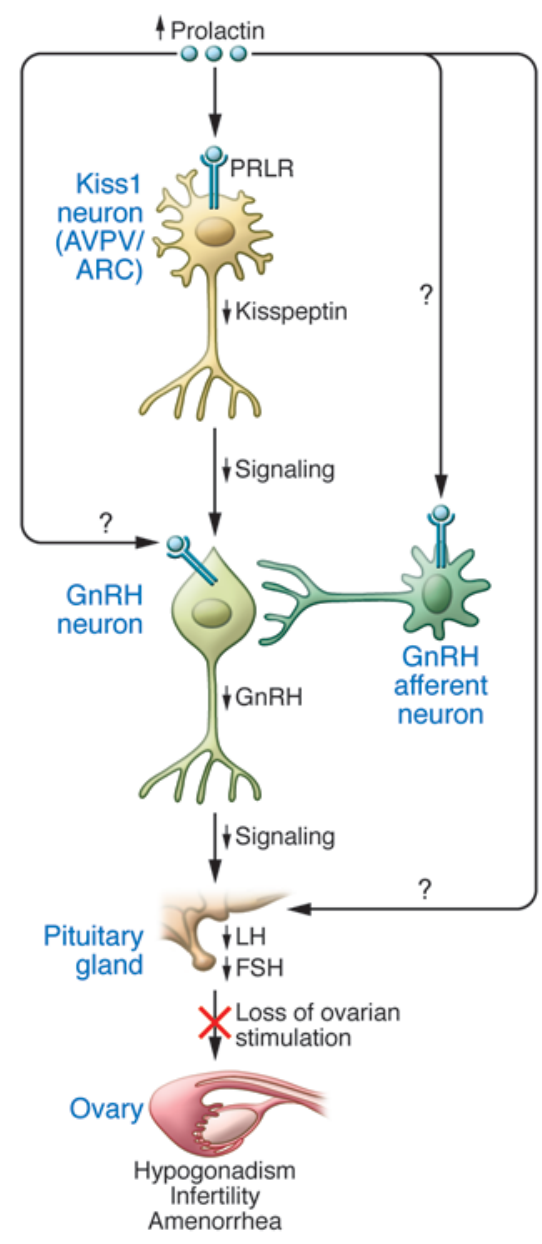

dopamine agonists to achieve normalization of prolactin levels and resumption of menstrual cycles remains the cornerstone for therapy of prolactinomas; this treatment addresses the underlying abnormality, and macroadenomas may enlarge during pregnancy if untreated $(1,2)$. Nonetheless, fertility induction with clomiphene citrate, gonadotropins, or pulsatile GnRH is an alternate approach for those patients with microprolactinomas who cannot tolerate dopamine agonists or for patients in whom dopamine agonists are not appropriate, such as in some instances of druginduced hyperprolactinemia. In this regard, it is striking that a single daily dose of kisspeptin was sufficient to restore ovulatory cycles in the mouse model. Coupled with recent evidence that kisspeptin infusion in humans can restore pulsatile LH secretion (8), treatment with kisspeptin or kisspeptin agonists may provide an avenue toward fertility induction in patients with hyperprolactinemia in the future.

\section{Acknowledgments}

I thank Le Min, Rona Carroll, and Wendy Kuohung for their critical reading of this report. This work was supported by the Eunice Kennedy Shriver National Institute of Child Health and Human Development, NIH, through cooperative agreement U54 HD28138 as part of the Specialized Cooperative Centers Program

\section{Figure 1}

Model of mechanisms of hyperprolactinemiainduced hypogonadism. Increased serum prolactin (PRL) levels result in decreased kisspeptin expression in Kiss1 neurons in both the hypothalamic arcuate (ARC) and anteroventral periventricular (AVPV) nuclei, mediated by prolactin receptors (PRLR) expressed on both populations of Kiss1 neurons. Suppression of kisspeptin, in turn, reduces $\mathrm{GnRH}$ release and results in loss of the ovulatory GnRH surge. This leads to reduced pituitary gonadotropin (LH and FSH) secretion and loss of ovarian stimulation, which results in hypogonadism, infertility, and amenorrhea. Prolactin may also have direct effects on GnRH neurons and/ or pituitary gonadotropes, or on other $\mathrm{GnRH}$ afferent neurons.

in Reproduction and Infertility Research; by R01 HD61577; and by R21 HD66495.

Address correspondence to: Ursula Kaiser, Brigham and Women's Hospital, 221 Longwood Avenue, Boston, Massachusetts 02115, USA. Phone: 617.525.8867; Fax: 617.264.5273; E-mail: UKaiser@ partners.org.

1. Melmed S, et al. Diagnosis and treatment of hyperprolactinemia: an Endocrine Society clinical practice guideline. J Clin Endocrinol Metab. 2011;96(2):273-288.

2. Schlechte JA. Long-term management of prolactinomas. J Clin Endocrinol Metab. 2007;92(8):2861-2865.

3. Evans WS, Cronin MJ, Thorner MO. Hypogonadism in hyperprolactinemia: Proposed mechanisms. In: Ganong WF, Martini L, eds. Frontiers In Neuroendocrinology. New York, New York, USA: Raven Press; 1982:77-122

4. Matsuzaki T, Azuma K, Irahara M, Yasui T, Aono T. Mechanism of anovulation in hyperprolactinemic amenorrhea determined by pulsatile gonadotropin-releasing hormone injection combined with human chorionic gonadotropin. Fertil Steril. 1994;62(6):1143-1149.

5. Kokay IC, Petersen SL, Grattan DR. Identification of prolactin-sensitive GABA and kisspeptin neurons in regions of the rat hypothalamus involved in the control of fertility. Endocrinology. 2011;152(2):526-535.

6. Sonigo C, et al. Hyperprolactinemia-induced ovarian acyclicity is reversed by kisspeptin administration. J Clin Invest. 2012;122(10):3791-3795.

7. Roa J, Navarro VM, Tena-Sempere M. Kisspeptins in reproductive biology: consensus knowledge and recent developments. Biol Reprod. 2011; 85(4):650-660.

8. George JT, et al. Kisspeptin-10 is a potent stimulator of LH and increases pulse frequency in men. JClin Endocrinol Metab. 2011;96(8):E1228-E1236. 